\title{
Gravitational Energy in Newtonian Gravity: A Response to Dewar and Weatherall
}

\author{
Patrick M. Duerr ${ }^{1}$. James Read ${ }^{2}$
}

Received: 17 December 2018 / Accepted: 25 September 2019 / Published online: 18 October 2019

(c) The Author(s) 2019

\begin{abstract}
The paper investigates the status of gravitational energy in Newtonian Gravity (NG), developing upon recent work by Dewar and Weatherall. The latter suggest that gravitational energy is a gauge quantity. This is potentially misleading: its gauge status crucially depends on the spacetime setting one adopts. In line with MøllerNielsen's plea for a motivational approach to symmetries, we supplement Dewar and Weatherall's work by discussing gravitational energy-stress in Newtonian spacetime, Galilean spacetime, Maxwell-Huygens spacetime, and Newton-Cartan Theory (NCT). Although we ultimately concur with Dewar and Weatherall that the notion of gravitational energy is problematic in NCT, our analysis goes beyond their work. The absence of an explicit definition of gravitational energy-stress in NCT somewhat detracts from the force of Dewar and Weatherall's argument. We fill this gap by examining the supposed gauge status of prima facie plausible candidates-NCT analogues of gravitational energy-stress pseudotensors, the Komar mass, and the Bel-Robinson tensor. Our paper further strengthens Dewar and Weatherall's results. In addition, it sheds more light upon the subtle link between sufficiently rich inertial structure and the definability of gravitational energy in NG.
\end{abstract}

Keywords Gravitational energy · Newtonian gravitation theory · Newton-Cartan theory $\cdot$ General relativity $\cdot$ Pseudotensors

$凶$ Patrick M. Duerr

patrick.duerr@oriel.ox.ac.uk

James Read

james.read@philosophy.ox.ac.uk

1 Oriel College, University of Oxford, Oxford, UK

2 Pembroke College, University of Oxford, Oxford, UK 


\section{Introduction}

Energy is a pivotal concept in all of physics. Ubiquitous - not least via the First Law of Thermodynamics - it has even been argued (by e.g. [7]) to be the salient metaphysical property of matter. It's therefore enticing to inquire into the status of the energy associated with the gravitational degrees of freedom in Newtonian Gravity (NG): Does NG admit of a meaningful definition of gravitational energy?

The question is of interest for at least three reasons. First, one would like to learn what makes gravity special, vis-à-vis other physical entities-perhaps already at the pre-general-relativistic level. Second, given that NG is an action-at-a-distance theory, one may wonder: Does this fact impinge upon the definition of a local notion of gravitational energy? With general relativity (GR), as a local field theory, in mind, one may ask: To what extent is NG free from the conceptual and interpretative difficulties of quasi-local notions of gravitational energy in GR (cf. e.g. [67])? Third, it's well known (e.g. [44], Ch. 12) that NG can be cast in a purely geometrical form, analogous to GR: In it, gravitational phenomena are re-conceptualised as manifestations of a nonflat spacetime geometry. This geometrisation seems to be linked to GR's notorious conceptual difficulties with respect to finding a meaningful notion of gravitational energy (cf. [48]). Echoing Bunge's suggestion, one might think, these difficulties in defining gravitational energy in GR intimate that gravity isn't a matter field, i.e. of the same type as the electromagnetic one (cf., for instance, Sotiriou, [66], Sect. 5.2). Studying NG in its geometrised form in closer detail thus promises to help us to better understand such conceptual difficulties - especially given that gravitational energy in NG's un-geometrised form is well-understood and unproblematic. Or so it appears.

In a recent paper [15], Dewar and Weatherall have challenged this. They assert that gravitational energy in Newtonian gravitational theories fails to be well-defined: that it's gauge-variant. The current paper responds to this claim. Notwithstanding our agreement with Dewar and Weatherall's overall conclusion, we feel that their reasoning leaves a few more things to be said-both formally and in substance. In particular, they don't attend to the question whether the spacetime setting makes a difference to the status of gravitational energy in NG. Furthermore, as Dewar and Weatherall (op.cit, p. 13) expressly acknowledge, their reasoning is predicated on a particular view of what counts as a gauge transformation. According to this view, Newton-Cartan Theory just is a gauge-invariant reformulation of NG. We explore the consequences of a different understanding gauge - one which, we contend, is closer to orthodoxy in the philosophy of physics literature (for better or worse).

We proceed as follows. Section 2 clarifies some preliminaries about gaugeinvariance and models (Sect. 2.1), and then reviews three non-geometrised classical spacetime settings for Newtonian Gravity: Newtonian spacetime (Sect. 2.2), Galilean spacetime (Sect. 2.3), and Maxwell-Huygens spacetime (Sect. 2.4); for each, the status of gravitational energy is assessed in detail. Section 3 focuses on Newton-Cartan Theory (NCT). Section 3.1 outlines NCT's basics. In Sect. 3.2, we explain why Dewar and Weatherall's objections against gravitational energy in NCT are specious. In Sect. 3.3, we try to fill the gap in their reasoning. Section 4 discusses the results achieved, and their relation to Dewar and Weatherall's own conclusions. 


\section{Gravitational Energy in Classical Spacetimes}

Dewar and Weatherall raise a deep question about the status of gravitational energy in Newtonian Gravity (NG): Is it a well-defined physical quantity? An answer isn't straightforward: Depending on which space-time setting one adopts, different variants of NG ensue [21, 43, 74]. As a result, the status of gravitational energy shouldn't be expected to be the same ab initio. After preliminaries about gauge-invariance (Sect. 2.1), this section reviews NG within the three non-geometrised classical space-time settings: Newtonian space-time (Sect. 2.2), Galilean space-time (Sect. 2.3) and Maxwell-Huygens space-time (Sect. 2.4), respectively.

\subsection{Models and Gauge-Invariance}

In this section, we clarify two concepts germane to the subsequent analysis: the classes of models relevant for us, and the notion of gauge equivalence.

Consider a given classical (non-quantum) theory $T$. Its associated models consist of $n$-tuples of geometrical objects, defined on a space-time manifold $\mathcal{M}$ (for details, see $[2,70])$. Some of these represent matter variables, e.g. particle positions or field configurations. The remaining objects represent space-time structure, encoding e.g. chronogeometric or inertial structure. ${ }^{1}$

The various space-time settings correspond to different choices for the space-time structure. Each choice ought to conform to Earman's adequacy condition: The spacetime symmetries should match the dynamical symmetries [19]. That is: The maximal group of diffeomorphisms under which the dynamical matter variables are invariant should coincide with those under which the spacetime structures are invariant. ${ }^{2}$ These matter variables are introduced as follows.

A theory's most general class of models is called its "kinematically possible models" (KPMs). According to Curiel [9], they specify two things.

1. A specification of the theory's ontology (in the sense of [55]): The KPMs individuate possible kinds of objects to which the theory $T$ is applicable-e.g. a viscous fluid or an electromagnetic field;

2. a specification of the theory's ideology (in the sense of Quine, ibid.): The KPMs enumerate (without determining) the degrees of freedom that form the complete state of possible objects of that kind at a point in time.

The laws which according to $T$ relate the entities in the KPMs are given by dynamical equations. In particular, these laws fix their law-like interrelations (e.g. diachronic evolution). They pick out of the KPMs the dynamically possible models (DPMs). (One may conceive of the KPMs as representing T's metaphysically possible worlds, say, of viscous fluids or electromagnetic fields. The DPMs describe nomologically possible worlds; in them, the laws of nature prescribed by $T$ hold. Models representing

\footnotetext{
1 We needn't embroil ourselves in the question of whether such a matter/space-time dichotomy can be upheld categorically (e.g. [6, 29, 35, 40, 42, 58, 60, 62]).

2 The transformation isn't to be applied to fixed fields in the theory. Here, a field is called 'fixed', if it is identically the same in every kinematically possible model (to be defined further below), cf. [4], fn 137.
} 
particular worlds - e.g. the actual one we inhabit—are obtained, if one further restricts the DPMs by boundary (or initial) conditions.)

Occasionally, one may wish to interpret a multiplicity of DPMs as representing the same world. This constitutes a gauge redundancy. On a mainstream view (which we won't call into question in this paper), ${ }^{3}$ for an object to represent a meaningful physical quantity, it must be gauge-independent. Else, it lacks intelligible identity conditions: The properties of an object violating gauge-independence are unclear.

The present paper isn't concerned with discussing the criteria of when to physically identify two models (cf. e.g. [12, 13, 27, 40, 45])—nor with the pondering on the question when to identify two theories (cf., for instance, [57]). While we won't critically discuss different positions on these matters, we'll nonetheless adopt a cautious stance. Regarding the identification of two models, we'll follow Møller-Nielsen's "motivational approach" (see below). Regarding the identity of gravitational theories, Dewar and Weatherall adopt the latter's own criterion [75]: Two empirically equivalent theories are merely reformulations of the same theory if they are categorically equivalent to one another. This stands in opposition to a more traditional view of theory equivalence, such as Glymour's [24, 25]. When discussing the variants of NG in the various space-time settings, we'll side with the received view: Contrary to Dewar and Weatherall, we'll treat them as different theories. To our minds, the absence of any consensus on such conundrums about theory equivalence (cf. [10, 32]) commends cautious conservatism. A given formalism can be interpreted in multiple ways. Whether it's to be regarded as equivalent to a theory couched in a different formalism depends on this interpretation.

Dewar and Weatherall aver that gravitational energy density in NG lacks gaugeindependence. This claim deserves scrutiny in each of the canonical non-geometric space-time settings. The remainder of the section will tackle this.

\subsection{Newtonian Spacetime}

Let's first consider NG set in Newtonian space-time (NST), $\mathrm{NG}_{\mathrm{NST}}$. Its KPMs consist of the 7-tuple

$$
\left\langle\mathcal{M}, t_{a b}, h^{a b}, \sigma^{a}, \nabla, \varphi, \rho\right\rangle
$$

Here, $\mathcal{M}$ denotes the smooth, 4-dimensional differentiable manifold of events in space-time.

$t_{a b}$ and $h^{a b}$ are smooth, symmetric tensor fields on $\mathcal{M}$, of signature $(1,0,0,0)$ and $(0,1,1,1)$, respectively. That is (see [39]): $\forall p \in \mathcal{M}: \exists\left(\xi_{(b)}^{a}\right)_{b=0, \ldots, 3} \in T \mathcal{M}$ such that $t_{a b} \xi_{(c)}^{a} \xi_{(d)}^{b}=\delta_{c, d} \delta_{c, 0}$.

Analoguously, for the spatial metric, $\forall p \in \mathcal{M}: \exists\left(\sigma_{b}^{(a)}\right)_{a=0, \ldots, 3} \in T^{*} \mathcal{M}$ such that $h^{a b} \sigma_{a}^{(c)} \sigma_{b}^{(d)}=\delta^{c, d}\left(\delta^{c, 1}+\delta^{c, 2}+\delta^{c, 3}\right)$. The two fields represent a temporal and a spatial "metric", respectively. Due to their degeneracy, they aren't metrics proper. In

$\overline{3 \text { Cf., however, Rovelli [61] for a discussion and a contrary position. }}$ 
particular, whilst being able to raise indices with $h^{a b}$, we can't lower them with an inverse metric. For these two metrics, the following three conditions hold ("orthogonality" and "temporal" and "spatial metric compatibility", respectively):

$$
\begin{gathered}
h^{a b} t_{b c}=0 \\
\nabla_{c} h^{a b}=0 \quad \& \quad \nabla_{c} t_{a b}=0 .
\end{gathered}
$$

Given a vector field $\xi^{a}$, its temporal length is defined via $\left(t_{b c} \xi^{b} \xi^{c}\right)^{1 / 2}$. The vector field is called time-like or space-like, if its temporal length is positive or zero, respectively. (For the analogous spatial "metric" we refer to [39]. The details subsequently play no important role.)

The vector field $\sigma^{a}$ is time-like (in the sense that $t_{a b} \sigma^{a} \neq 0$ ). Its integral curves represent the persisting points of absolute space. It grounds a standard of absolute rest/motion.

$\nabla$ is a flat derivative operator on $\mathcal{M}^{4}$ :

$$
\mathrm{R}_{b c d}^{a}=0 .
$$

It supplies the geodesic/inertial structure-loosely speaking: a standard of straightness-in terms of which inertial motion is defined. ${ }^{5}$

The gravitational potential and its source, the mass density, are represented by the smooth scalar fields $\varphi$ and $\rho$. (For simplicity, we'll ignore in the following trivial gauge transformations of the potential, $\varphi \mapsto \varphi+\varphi_{0}$, for constant $\varphi_{0} \cdot{ }^{6}$ )

Throughout, we'll assume that classical space-times are temporally orientable. That is: There exists a continuous, globally defined covector field $t_{a}$ such that $t_{a b}=t_{a} t_{b}$. A time-like vector $\xi^{a}$ is future-directed, if $\xi^{a} t_{a}>0$. Otherwise, it's past-directed. In conjunction with the orthogonality and metric compatibility conditions, orientability allows us to slice up a spacetime into simultaneity hypersurfaces (see [39]).

In DPMs of $\mathrm{NG}_{\mathrm{NST}}$, the gravitational potential $\varphi$ obeys the Newton-Poisson Equation,

$$
h^{a b} \nabla_{a} \nabla_{b} \varphi=4 \pi \rho .
$$

Consider now Galilean (static and kinematic) shifts ${ }^{7}$ :

$$
\Gamma:\left\{\begin{array} { c } 
{ t } \\
{ x ^ { i } }
\end{array} \mapsto \left\{\begin{array}{c}
t+t_{0} \\
x_{0}+R_{j}^{i} x^{j}+v^{i} t
\end{array}\right.\right.
$$

${ }^{4}$ Recall that this means that the concatenation of parallel transporting a vector $\xi^{a}$ commutes:

$$
\nabla_{[a} \nabla_{b]} \xi^{c} \equiv R_{d a b}^{c} \xi^{d}=0 .
$$

5 It deserves to be underlined that the role of inertial structure isn't exhausted by explaining (or grounding) inertial motion (see e.g. [54], Sect. 5.2).

6 Strictly speaking, in order for this shift to be regard as trivial, one must embrace a form of anti-quidditism about properties. See Martens and Read [41], for details.

7 This terminology follows Huggett [31]. 
They comprise uniform time $\left(t_{0}\right)$ and space translations $\left(x_{0}\right)$, time-independent spatial rotations $\left(R_{j}^{i}\right)$, and constant velocity boosts $\left(t v^{i}\right)$ in absolute space. In geometric terms this translates into linear transformations of the type $\sigma^{a} \mapsto S_{b}^{a} \sigma^{b}+\sigma_{0}^{a}$ for a constant vector field $\sigma_{0}^{a}$ and a constant orthogonal matrix $S_{b}^{a}$ with $\operatorname{det}\left(S_{b}^{a}\right)=1$ ([19], Ch. 2).

In $\mathrm{NG}_{\mathrm{NST}}$, kinematic shifts reflect meaningful differences. (Throughout, we'll adopt the position known as sophisticated substantivalism, see e.g. [54]. It denies that static shifts - uniform time and space translations - correspond to physically distinct possibilities.) They describe distinct, velocity-boosted worlds. A material reference body in kinematically shifted models moves at different velocities $v^{i}$ relative to the persisting points of absolute space.

Already Newton himself, in his Corollary $\mathrm{V},{ }^{8}$ acknowledged the symmetry of models of $\mathrm{NG}_{\mathrm{NST}}$ under Galilean shifts: Its laws remain invariant under them; with respect to the laws, Galilei-shifted models are indistinguishable.

Dynamical shifts generalise kinematic ones. They allow for an arbitrary timedependent translation $\vec{d}(t)$, concomitant with a re-scaling of the potential:

$$
\Delta:\left\{\begin{array} { l } 
{ \vec { x } } \\
{ \varphi }
\end{array} \mapsto \left\{\begin{array}{c}
\vec{x}+\vec{d}(t) \\
\varphi-\ddot{\vec{d}} \cdot \vec{x}+f(t) .
\end{array}\right.\right.
$$

In a dynamical shift, one subjects the system to a uniform acceleration, $\ddot{\vec{d}}(t)$, and adds a force that remains constant on simultaneity surfaces. (The question of how to translate this into the coordinate-free language of differential geometry needn't distract us here; we'll return to it in Sect. 3.1). A fortiori, dynamical shifts mediate meaningful differences: They represent universes in which some material reference body moves at different (uniform) accelerations with respect to the persisting points of absolute space.

Two models of $\mathrm{NG}_{\mathrm{NST}}$, related via dynamical shifts, thus also represent distinct worlds. ${ }^{9}$ (We'll see presently that they are nonetheless observationally indiscernible.)

According to Dewar and Weatherall, dynamical shifts threaten the gauge-invariance of gravitational energy. To see how, let's first introduce the energy density of the gravi-

\footnotetext{
8 "The motions of bodies included in a given space are the same among themselves, whether that space is at rest, or moves uniformly forwards in a right line without any circular motion" (Newton 1729).

9 We'll set aside here the question of whether dynamically shifted models still constitute solutions of $\mathrm{NG}_{\mathrm{NST}}$. Potential doubts might arise from the fact that the "sourceless sources", driving such shifts, are inimical to the Newtonian framework. At least as it stands, this argument doesn't sway us. First, the historical Newtonian framework has no direct bearing on the systematic question at hand. Secondly, and more importantly, to assess the question from a systematic angle, one must spell out what one means by, and what is included in the "Newtonian framework". An explicit argument must then be given why "sourceless sources" are indeed prohibited within it. (For instance, it's not obvious that the Newton's Third Law is applicable: It refers only to forces - and one may deny that dynamical shifts constitute forces proper. With forces being arguably causes, we have hereby touched on a subtle question in the metaphysics of causation within Newtonian physics.) Given the lack of a robust consensus on the details of the metaphysical framework appropriate to Newtonian physics—and the ineluctable disputes concomitant with metaphysical frameworks quite general—we deem it prudent to remain neutral on whether dynamical shifts don't preserve solutions of $\mathrm{NG}_{\mathrm{NST}}$. We thank an anonymous referee for pressing us on this important subtlety.
} 
tational potential $\varphi$ as the Noether-current associated with time-translation invariance of the NG Lagrangian,

$$
E^{\left(N G_{N S T}\right)}=-\frac{1}{8 \pi} h^{a b} \nabla_{a} \varphi \nabla_{b} \varphi
$$

It's easily verified to be invariant exactly under Galilei-shifts: Static and kinematic shifts don't alter the gravitational energy density. By contrast, due to the scaling in the gravitational potentials, dynamical shifts do: Two $\mathrm{NG}_{\mathrm{NST}}$ models related via dynamical shifts differ on their gravitational energy density.

Should this disconcert us? Clearly-no: The two NST models, related via kinematic $(\Gamma)$ or dynamic shifts $(\Delta)$ describe distinct worlds. Consequently, gravitational energy density, set within NST, isn't a gauge-quantity - contra Dewar and Weatherall. That gravitational energy doesn't vary between such worlds is irrelevant.

In conclusion: NST has sufficient structure to ward off the spectre of gaugedependence for gravitational energy density.

To be sure, NST is an objectionable space-time setting for NG. Via its unobservable absolute standard of rest, its dynamical and space-time symmetries don't align. This flouts Earman's adequacy conditions. Yet, one mustn't conflate the flaws of a space-time setting with the (alleged) shortcomings of a quantity-gravitational energy-defined within this space-time setting.

How does the situation look in space-time settings that amend this defect of NST? We next discuss Galilean space-time.

\subsection{Galilean Space-Time (GST)}

GST ameliorates (some of) NST's shortcomings: It drops the assumption of absolute rest, i.e. the vector field $\sigma^{a}$. Thereby, one can pare down redundant structure. The points of space in GST no longer persist: Their diachronic identity is jettisoned. In $\mathrm{NG}_{\mathrm{GST}}$, one identifies all DPMs of $\mathrm{NG}_{\mathrm{NST}}$ that differ only through Galilean shifts $\Gamma .{ }^{10}$ Thus, GST retains an absolute standard of straightness of paths between two events: Whether a path is straight-a geodesic with respect to the flat derivative operator $\nabla_{b}$-is an absolute matter of fact. (In modal language: Those DPMs of $\mathrm{NG}_{\mathrm{NST}}$ in which the spatio-temporal paths of all possible test matter are parallel are identified as describing the same world.) ${ }^{11}$ Contrariwise, as in NST, dynamically shifted DPMs remain distinct: In $\mathrm{NG}_{\mathrm{GST}}$, dynamical shifts aren't gauge-transformations.

\footnotetext{
${ }^{10}$ In the case of static shifts, as discussed above, one may invoke sophisticated substantivalism, i.e. the denial that worlds are distinct that differ only with regard to which spacetime points exhibit which metrical properties (cf., for instance, Pooley ([54], Sect. 7).

In the case of kinematic shifts, the symmetry arguably only motivates the search for a more perspicuous ontology that can metaphysically elucidate the identity of kinematically shifted worlds [46]. This is provided by GST's transition from a 3-dimensional to the 4-dimensional picture of reality (cf., for instance, [43], pp. 54).

11 This follows from the fact that the totality of geodesics on a manifold uniquely determine a derivative operator.
} 
What does this imply for gravitational energy density? The expression for gravitational energy density for $\mathrm{NG}_{\mathrm{GST}}$ carries over from Sect. 2.3:

$$
E^{\left(N G_{G S T}\right)}=-\frac{1}{8 \pi} h^{a b} \nabla_{a} \varphi \nabla_{b} \varphi .
$$

It didn't depend on $\sigma^{a}$, anyway. Under dynamical shifts it changes.

But as before, this is harmless: Dynamically shifted DPMs describe distinct worlds. Hence, that they differ on their gravitational energy, doesn't render the latter gaugevariant. (To be sure, dynamically shifted DPMs are empirically indistinguishable. All relational quantities remain unaltered. So, an observer in one of several dynamically shifted worlds couldn't ascertain which is hers. This predicament may be metaphysically lamentable - but it's not a shortcoming of gravitational energy.)

As in the $\mathrm{NG}_{\mathrm{NST}}$ case, Dewar and Weatherall's diagnosis of the gauge-dependence of gravitational energy is therefore unfounded in $\mathrm{NG}_{\mathrm{GST}}$.

The rebuttals of Dewar and Weatherall's claim so far may appear trivial. After all, GST - and a fortiori NST - arguably aren't the most perspicuous space-time settings for NG. ${ }^{12}$ The analysis becomes more interesting for the two possible improvements on $\mathrm{NG}_{\mathrm{GST}}$, Maxwell-Huygens Gravity (NGMHST) and Newton-Cartan Theory (NCT), respectively. We'll conclude this section with the former, before turning to the latter in Sect. 3.

\subsection{Maxwell-Huygens Spacetime (MHST)}

In light of his Corollary VI, ${ }^{13}$ Newton may be credited with recognising the empirical indistinguishability of models of NG related via uniform accelerations, $\vec{x} \mapsto \vec{x}^{\prime}=\vec{x}+\vec{d}$ ( $t$ ) [64]. Here, $\vec{d}$ is a twice-differentiable function, representing an accelerational boost. Nonetheless, Newton persevered in his belief in absolute space. In rational (albeit historically incorrect, see Hoefer and Huggett [30], Sect. 6; [63]) reconstructions, he is frequently (e.g. [43], Ch. 2) imputed an invocation of an inference to the best explanation for inertial effects. Consider, for instance, the surface of a water-filled pail. It's (observably!) concave, if and only if the bucket is rotating. Is this rotation best conceptualised as rotation in absolute space, with the latter understood at least at the level of $\mathrm{NG}_{\mathrm{GST}}$ ? At first blush, it might appear so. But in fact, $\mathrm{NG}_{\mathrm{MHST}}$ further whittles down $\mathrm{NG}_{\mathrm{GST}}$ 's structure by exploiting the symmetry of the Poisson Equation under uniform accelerations. $\mathrm{NG}_{\mathrm{GST}}$ only preserves an absolute sense of non-linear acceleration (equivalently ${ }^{14}$ : rotation), evinced in inertial effects, such as in the above bucket experiment.

\footnotetext{
12 It's all the more surprising that GST is the only space-time setting (apart from NCT) for NG which Dewar and Weatherall explicitly consider.

13 "If bodies, anyhow moved among themselves, are urged in the direction of parallel lines by equal accelerative forces; they will all continue to move among themselves, after the same manner as if they had been urged by no such forces" (Newton 1729).

14 To be sure: A time-dependent linear acceleration - a linear acceleration that is still an arbitrary function of time, i.e. of the form $\xi^{b} \nabla_{b} \xi^{a}=\alpha^{a}(t)+\beta(t) \xi^{a}$-isn't equivalent to a rotation. But any non-linear acceleration is.
} 
In $\mathrm{NG}_{\mathrm{MHST}}$, as we understand it (see below), one identifies $\mathrm{NG}_{\mathrm{GST}}$ models related via uniform accelerations ${ }^{15}$ : One stipulates that they describe the same world. In contrast to uniform accelerations in NST and GST, in MHST they are gaugetransformations.

Recall that within the geometric framework of classical space-times, derivative operators encode inertial structure. Hence, they define standards of accelerations. For MHST, one must thus identify those derivative operators that correspond to the same standards of non-rotational acceleration, up to uniform-accelerational transformations. This translates into the following condition for any two such standards of acceleration $\nabla$ and $\nabla^{\prime}$, and all unit, time-like vector-fields $\xi^{a}$ (see [39] for details):

$$
\nabla^{[a} \xi^{b]}=0 \Leftrightarrow \nabla^{\prime[a} \xi^{b]}=0 .
$$

One can envision this condition as the requirement that the verdict whether trajectories of free particles in those space-times are not twisted be independent of the choice of the standard of acceleration. Such space-times are rotationally equivalent, with rotation understood as the twisting of possible free-fall trajectories.

Formally, one can now quotient out those of $\mathrm{NG}_{\mathrm{GST}}$ 's KPMs which differ only by uniform accelerations (for details, see [73]). A KPM in $\mathrm{NG}_{\mathrm{MHST}}$ thus takes the form of the following 6-tuple:

$$
\left\langle\mathcal{M}, t_{a}, h^{a b},[\nabla], \varphi, \rho\right\rangle \text {. }
$$

The only novel object, unfamiliar from GST is [ $\nabla]$, the "standard of rotation". It's the equivalence class of flat, metrically compatible, rotationally equivalent derivative operators in $\mathrm{GST}^{16}$ :

$$
[\nabla]=\left\{\nabla^{\prime}: \mathrm{R}_{b c d}^{\prime a}=0 \& \nabla_{a}^{\prime} t_{b} \& \nabla_{c}^{\prime} h^{a b}=0 \&\left[\left|\xi^{a} t_{a}\right|=1 \Rightarrow\left(\nabla^{\prime}\left[a \xi^{b]}=0 \Leftrightarrow \nabla^{[a} \xi^{b]}=0\right)\right]\right\}\right.
$$

Here, $\mathrm{R}_{b c d}^{\prime a}$ is the Riemann tensor associated with $\nabla^{\prime} .{ }^{17}$

It's straightforward to show that the Newton-Poisson Equation in GST, $h^{a b} \nabla_{a} \nabla_{b} \varphi=4 \pi \rho$, remains invariant under changes of rotationally equivalent derivative operators [14]. DPMs in $\mathrm{NG}_{\mathrm{MHST}}$ can thus be obtained by identifying rotationally equivalent, but otherwise identical, DPMs of $\mathrm{NG}_{\mathrm{GST}}$.

To invest $\mathrm{NG}_{\mathrm{MHST}}$ with empirical content, we still need equations of motion for matter under the influence of gravity. They group together the equations of motion for $\mathrm{NG}_{\mathrm{GST}}$ within a standard of rotation, such that their (time-like, unit) solutions

\footnotetext{
15 Again, considering the Poisson equation only. Particle equations of motion will be considered below.

16 Speaking of an equivalence class of derivative operators, rather than (e.g.) defining a primitive standard of rotation (as does e.g. Weatherall [73]), invokes Dewar's 'sophistication' about symmetries [14]. A recent skeptical attitude towards said 'sophistication' can be found in Martens and Read [41]. Since we share the latters' skepticism, ultimately we would find it preferable to work with Weatherall's standard of rotation, rather than an equivalence class of operators. Nevertheless, for continuity with the literature, we set such concerns aside in the remainder of this article.

17 Two rotationally equivalent derivative operators $\nabla$ and $\nabla^{\prime}$ in this class are related via $\nabla^{\prime}=\left(\nabla, \eta^{a} t_{b} t_{c}\right)$ for some spacelike vector field $\eta^{a}$, satisfying $\nabla^{b} \eta^{a}=0$ [14].
} 
$\xi, \xi^{\prime} \in T \mathcal{M}$ define accelerations (with respect to the same derivative operators) that differ only by linear accelerations ${ }^{18}$ :

$$
\left\{\xi \in T \mathcal{M}: \exists \nabla^{\prime} \in[\nabla] \text { such that } \xi^{b} \nabla_{b}^{\prime} \xi^{a}+\nabla^{\prime a} \varphi=0\right\}=\bigcup_{\nabla^{\prime} \in[\nabla]}\left\{\xi \in T \mathcal{M}: \xi^{b} \nabla_{b}^{\prime} \xi^{a}+\nabla^{\prime a} \varphi=0\right\} .
$$

The (class of) equations of motion picking out this solution set, $\left\{\xi^{b} \nabla^{\prime}{ }_{b} \xi^{a}+\nabla^{a} \varphi=0: \nabla^{\prime} \in[\nabla]\right\}$, is trivially invariant under uniform accelerations.

What might be candidates for gravitational energy in $\mathrm{NG}_{\mathrm{MHST}}, E^{\left(N G_{\mathrm{MHST}}\right)}$ ? The most natural one is defined as the equivalence class of all gravitational energy densities of rotationally equivalent $\mathrm{NG}_{\mathrm{GST}}$ models. As the action of two derivative operators upon a scalar is the same, $\nabla \varphi=\nabla^{\prime} \varphi$, this equivalence class is well-defined: The gravitational energy densities of two Galilean spacetimes with rotationally equivalent derivative operators $\nabla^{\prime}$ and $\nabla$ coincide,

$$
E^{\prime}=-\frac{1}{8 \pi} h^{a b} \nabla_{a}^{\prime} \varphi \nabla^{\prime}{ }_{b} \varphi=-\frac{1}{8 \pi} h^{a b} \nabla_{a} \varphi \nabla_{b} \varphi=E .
$$

Consequently, within a model of $\mathrm{NG}_{\mathrm{MHST}}$, gravitational energy density is a welldefined quantity. It's not gauge-dependent. ${ }^{19}$ (Note that while uniform accelerations are gauge transformations in $\mathrm{NG}_{\mathrm{MHST}}$, dynamical shifts, which also include a transformation of the potential, aren't.)

Again, Dewar and Weatherall's proclamation of the gauge-dependence of gravitational energy doesn't apply to $\mathrm{NG}_{\mathrm{MHST}}$. Like in $\mathrm{NG}_{\mathrm{GST}}$, that dynamically shifted models of $\mathrm{NG}_{\mathrm{MHST}}$ differ in their gravitational energy densities is benign: They describe distinct worlds.

One may, however, repudiate this formulation of NG within MHST for two reasons: its implausible conceptual prerequisites and its radicalness, respectively.

Firstly, it's unsatisfactory that in order to define MHST via an equivalence class of derivative operators of GST, one draws on structure that ultimately one doesn't attribute to the space-time (cf. Dewar [13], Martens and Read [41], Weatherall [73]). ${ }^{20}$

Compare the transition from NST to GST: There, the standard of absolute rest, represented by the time-like vector field $\sigma^{a}$, could simply be excised: It played only an otiose role in the formulation of NG.) Indeed, elsewhere Weatherall [73] proffers an alternative characterisation of MHST without reference to derivative operators. Absent

\footnotetext{
18 We wish to underscore that, given our cautious approach to theory identity, our version of $\mathrm{NG}_{\mathrm{MHST}}$ differs from the ones, primarily considered in the literature (e.g. [14]; Weatherall [73]). Our version's ontology includes a gravitational field $\nabla^{a} \varphi$. By contrast, in Weatherall's [73] treatment, "we (do not) need to interpret the gravitational potential or corresponding gravitational field, $\nabla \varphi$, as representing facts about force or a field-like entity" (p. 88). Similarly, in Dewar's treatment there is also no (privileged) choice of gravitational potential or gravitational field.

We thank an anonymous referee for pressing us on this.

19 Note that on Dewar's version of $\mathrm{NG}_{\text {MHST }}$ the gravitational energy density would count as gauge-variant, as the potential would change.

20 One could also rephrase this objection in terms of physical degrees of freedom. Concepts natural to a theory reflect these, as it were, carving nature at its joints. For NCT, the true physical quantities are $U$ (1)-invariants - rather than boosts, parameterized by $U$ (1) [68].
} 
a derivative operator, though, how to define gravitational energy density? Evidently, the standard definition is no longer available in that case.

Gravitational energy density could well turn out not to be definable at all (as Dewar and Weatherall themselves admit)! That would certainly be grist to Dewar and Weatherall's mills - but for reasons other than those they cite. (It would be desirable to investigate whether gravitational energy density could be defined without derivative operators. We'll not pursue this, here, though.)

Elsewhere, Weatherall ([73], Sect. 5) draws attention also to a second blemish of MHST: It's more revisionary than at first blush it appears. Forces as they figure on the 1.h.s. of Newton's 2nd Law are absolute: They are formulated in terms of one derivative operator. It's unclear whether all of (non-gravitational) physics can be reformulated on MHST. Think, for instance, of the Abraham-Lorentz-Dirac force, describing the recoil force of accelerated charged particles due to radiation (see [59]): It's manifestly not invariant under uniform accelerations. Hence, adopting MHST as the space-time setting for Newtonian physics necessitates a revision of the mathematical and conceptual foundations of much of classical physics. This may seem gratuitously radical. ${ }^{21}$

Another response to $\mathrm{NG}_{\mathrm{GST}}$ 's redundancy is therefore appealing. In conjunction with the equivalence of inertial and gravitational mass, its symmetry under dynamical shifts motivates a geometrisation of NG: Like in General Relativity, gravitational effects are absorbed into the space-time's non-flat inertial structure. The result is known as Newton-Cartan Theory (NCT). To this we turn next.

\section{Newton-Cartan Theory}

We'll now investigate Dewar and Weatherall's claim that gravitational energy isn't well-defined in NCT. This section first (Sect. 3.1) reviews the basics of NCT. Next (Sect. 3.2), we expound why Dewar and Weatherall's arguments are specious. In Sect. 3.3, we try to fill the gap in their reasoning.

\subsection{Geometrised NG}

In this section, we review the basics of NCT, as contained in Trautman's Geometrisation Lemma and its converse Recovery Theorem (for all details, see [39], Ch. 4.2).

In NCT, the gravitational potential of NG is absorbed into NCT's (non-flat) derivative operator. This is encapsulated in Trautman's Geometrisation Lemma.

Let $\left\langle\mathcal{M}, t_{a}, h^{a b}, \nabla_{a}\right\rangle$ be a Galilean (henceforth: "classical") spacetime. (The derivative operator $\nabla_{a}$ is assumed to be flat; its associated Riemann tensor vanishes, $\mathrm{R}_{b c d}^{a}=0$.) Let furthermore $\varphi$ and $\rho$ be smooth, real-value scalar fields on $\mathcal{M}$ which

\footnotetext{
21 The revisionary nature of MHST also crops up with respect to its interpretation. Recall that (considering the gravitational field equations only) two GST models correspond to the same Maxwell-Huygens spacetime, if and only if they differ merely up to uniform accelerations. Consequently, two DPMs of GST that, albeit rotationally equivalent, differ merely in their potentials, count as distinct. That raises the question of how to interpret the scalar in MHST: What is its ontological status? Is it a real physical field, on a par with, say, the electromagnetic one? On which space does it live?
} 
obey the Poisson Equation, $h^{a b} \nabla_{a} \nabla_{b} \varphi=4 \pi \rho$. Finally, let $\tilde{\nabla}_{a}=\left(\nabla,-\mathrm{t}_{a} \mathrm{t}_{b} h^{c d} \nabla_{d} \varphi\right) .{ }^{22}$ Then, the following three propositions hold:

1. $\left\langle\mathcal{M}, t_{a}, h^{a b}, \tilde{\nabla}_{a}\right\rangle$ is a classical spacetime.

2. $\tilde{\nabla}_{a}$ is the (unique) derivative operator such that for all time-like curves on $\mathcal{M}$ with 4-velocity $\xi^{a}: \xi^{a} \tilde{\nabla}_{a} \xi^{b}=0 \Leftrightarrow \xi^{a} \nabla_{a} \xi^{b}=-h^{b c} \nabla_{c} \varphi$.

3. The Riemann curvature $\tilde{\mathrm{R}}_{b c d}^{a}$ associated with $\tilde{\nabla}_{a}$ satisfies the

a. the "geometrised" Poisson Equation $\tilde{R}_{a b}:=\tilde{\mathrm{R}}_{a c b}^{c}=4 \pi \rho t_{a} t_{a}$,

b. and the curvature conditions $\tilde{\mathrm{R}}_{c d}^{a b}=0 \& \tilde{\mathrm{R}}_{b d}^{a c}=\tilde{R}_{d b}^{c a}$.

The second proposition states an equivalence between geodesic/unaccelerated/inertial motion with respect to one derivative operator, and particular accelerated/non-inertial motion with respect to another: Exactly those curves are geodesics with respect to $\tilde{\nabla}_{a}$ that describe accelerated motion that is the result of the Newtonian gravitational force, with respect to $\nabla_{a}$. In this sense gravity is geometrised—or rather "inertialised" (cf. [47], Ch. 9; [38], esp. §4): The deviation from inertial trajectories, defined via $\nabla_{a}$, due to the gravitational force is reconceptualised as a manifestation of (non-flat) inertial structure, defined via $\tilde{\nabla}_{a}$. (The interpretation of the curvature conditions shan't concern us here. Instead, we refer to [39], Ch. 4.3.)

Via the Recovery Theorem, we can re-translate geometrised NCT gravity back into non-geometrised $\mathrm{NG}_{\mathrm{GST}}$.

Let the classical spacetime $\left\langle\mathcal{M}, t_{a}, h^{a b}, \tilde{\nabla}_{a}\right\rangle$ satisfy the geometrised Poisson Equation $\tilde{\mathrm{R}}_{a b}=4 \pi \rho t_{a} t_{a}$ for some smooth scalar field $\rho$ on $\mathcal{M}$, and the Trautmann curvature conditions $\tilde{\mathrm{R}}_{c d}^{a b}=0 \& \tilde{\mathrm{R}}_{b d}^{a c}=\tilde{R}_{d b}^{c a}$. Then, in the neighbourhood of any point a realvalued scalar $\varphi$ and a derivative operator $\nabla$ exist, such that the following propositions hold:

1. $\nabla$ is compatible with $t_{a}$ and $h^{a b}$.

2. $\nabla$ is flat. (Its associated Riemann tensor vanishes, $\mathrm{R}_{b c d}^{a}=0$.)

3. For all time-like curves on $\mathcal{M}$ with 4-velocity $\xi^{a}: \xi^{a} \tilde{\nabla}_{a} \xi^{b}=0 \Leftrightarrow \xi^{a} \nabla_{a} \xi^{b}=$ $-h^{b c} \nabla_{c} \varphi$.

4. $\varphi$ satisfies the Poisson Equation: $h^{a b} \nabla_{a} \nabla_{b} \varphi=4 \pi \rho$.

Via the Recovery Theorem, we can "de-geometrise" NCT spacetimes: Geodesic/inertial motion with respect to $\tilde{\nabla}$, which was force-free, is now reconceptualised as accelerated/non-inertial motion with respect to $\nabla$, subject to the gravitational force.

The de-geometrisation isn't unique. A second pair $\varphi^{\prime}$ and $\nabla^{\prime}$ for which

$$
h^{a b} \nabla_{a} \nabla_{b}\left(\varphi-\varphi^{\prime}\right)=0 \quad \& \quad \nabla^{\prime}=\left(\nabla, \mathrm{t}_{a} \mathrm{t}_{b} h^{c d} \nabla_{d}\left(\varphi-\varphi^{\prime}\right)\right)
$$

also satisfies the conditions $1-4$ of the Recovery Theorem.

\footnotetext{
22 That is (see [39], Ch. 1.7): Let $\nabla$ and $\nabla^{\prime}$ be two derivative operators on a manifold $\mathcal{M}$. Then, they are uniquely related via a symmetric tensor field $C_{b c}^{a}$ : For any tensor $\alpha_{b_{1} \ldots b_{s}}^{a_{1} \ldots a_{r}}$ of rank $(r, s)$ on $\mathcal{M}$, $\left(\nabla_{m}^{\prime}-\nabla_{m}\right) \alpha_{b_{1} \ldots b_{s}}^{a_{1} \ldots a_{r}}=\alpha_{n b_{2} \ldots b_{s}}^{a_{1} \ldots a_{r}} C_{m b_{1}}^{n}+\alpha_{b_{1} n b_{3} \ldots b_{s}}^{a_{1} \ldots a_{r}} C_{m b_{3}}^{n}+\cdots-\alpha_{b_{1} \ldots b_{s}}^{d a_{2} \ldots a_{r}} C_{m d}^{a_{1}}-\alpha_{b_{1} \ldots b_{s}}^{a_{1} d a_{3} \ldots a_{r}} C_{m d}^{a_{2}}-\cdots$
} 
The transformations between any pair $(\varphi, \nabla)$ and $\left(\varphi^{\prime}, \nabla^{\prime}\right)$ that each satisfies the two non-uniqueness conditions are the dynamical shifts, mentioned in Sect. 2.2. Consequently, two models of $\mathrm{NG}_{\mathrm{GST}}$ related via dynamical shifts are "de-geometrisations" of the same NCT spacetime. It has therefore been argued-e.g. by Pooley ([54], Sect. 6.1.1) or Knox [34] - that the gravitational scalar and the derivative operator of ungeometrised $\mathrm{NG}$-i.e. $\mathrm{NG}_{\mathrm{GST}}$ - are merely gauge-dependent quantities; geometrised NG-i.e. NCT-provides a gauge-free formulation of NG. With its dynamical symmetries matching its spacetime symmetries, and hence conforming to Earman's adequacy conditions, NCT is a satisfactory theory of gravity.

In summary: NCT allows us to re-conceptualise gravitational effects as manifestations of non-flat spacetime geometry (inertial structure). Models of $\mathrm{NG}_{\mathrm{GST}}$ related via dynamical shifts can be identified as the same NCT spacetime.

\subsection{Dewar and Weatherall on Gravitational Energy in NCT}

Let's now assess Dewar and Weatherall's principal argument against gravitational energy in NCT. Its logical form can be reconstructed as follows:

(1) The natural expression for gravitational energy in $\mathrm{NG}_{\mathrm{GST}}$ isn't invariant under dynamical shifts.

(2) In NCT, one identifies those DPMs of $\mathrm{NG}_{\mathrm{GST}}$ that are related via dynamical shifts as physically equivalent; they are gauge.

(3) Therefore, gravitational energy in NCT isn't gauge-invariant.

Our authors correctly observe (1) and (2). However, their conclusion-(3) - is objectionable for a simple reason: Nowhere do Dewar and Weatherall explicitly define the object that is supposed to most naturally represent gravitational energy in NCT.

This is a crucial shortcoming. It renders their argument both formally and substantively incomplete. After all, Trautman's Geometrisation Lemma and Recovery Theorem (Sect. 3.1) only equip us with a translation between the 6-tuple $\left\langle\mathcal{M}, t_{a}, h^{a b}, \nabla_{a}, \rho, \varphi\right\rangle$ of non-geometrised $\mathrm{NG}_{\mathrm{GST}}$ quantities, and the 5-tuple $\left\langle\mathcal{M}, t_{a}, h^{a b}, \tilde{\nabla}_{a}, \rho\right\rangle$ of geometrised NCT quantities; both are silent on any other quantities.

For Dewar and Weatherall's above syllogism to formally go through, premise (1) needs to be superseded by

(1') The (most natural) NCT counterpart of the Galilean gravitational energy isn't invariant under dynamical shifts.

With this, the conjunction of all three premises entails the conclusion:

$$
\left(1^{\prime}\right) \&(2) \rightarrow(3)
$$

But why believe that (1') is true? It's far from clear-as Dewar and Weatherall concede themselves - whether the NCT counterpart of Galilean gravitational energy even exists - and if it does, whether it indeed fails to be invariant under dynamical shifts. 
(To be sure, if either could be negated, this would be grist to Dewar and Weatherall's mills. Their conclusion would remain intact. But it would follow from different reasons: namely those against the existence of the most natural NCT counterpart of Galilean gravitational energy, rather than the gauge-dependence of an actually existing NCT gravitational energy.) In short: It's one thing to doubt the definability of gravitational energy; it's another to doubt its physical meaningfulness (or well-definedness). Dewar and Weatherall focus on the latter.

Even if one charitably grants that the meaning of "most natural candidate" is clear, one may impugn the very existence of an NCT counterpart of Galilean gravitational energy. As the Geometrisation Lemma discloses, Galilean gravitational energy contains terms absent in NCT. In the latter's DPMs, a gravitational potential doesn't appear; it has been absorbed by NCT's non-flat connection. Furthermore, Galilean gravitational energy is defined via the (flat) derivative operator of GST. Which derivative operator should then enter the NCT counterpart of Galilean gravitational energy? An intuitive choice would, of course, be NCT's (non-flat) derivative operator. But this is scarcely compelling.

If thus gravitational energy in $\mathrm{NG}_{\mathrm{GST}}$ essentially hinges on terms absent in $\mathrm{NCT}$, then why assume that it can be defined at all in NCT?

In conclusion: Unless the possible candidate for NCT's gravitational energy is explicitly defined, Dewar and Weatherall's criticism of the latter's (alleged) gaugedependence forfeits much of its force.

To fill this lacuna, we'll now discuss various concrete options.

\subsection{Candidates for Gravitational Energy in NCT}

In the preceding section, we argued that Dewar and Weatherall's criticism of gravitational energy in NCT is vitiated by their lack of an explicit definition of gravitational energy in NCT. Here, we'll examine a number of natural candidates: 1. Pseudotensors, 2. Komar energy, 3. Lorentz and Levi-Civita's proposal, 4. The Bel-Robinson tensor. 5. Pittsification. Rather than suffering from gauge-dependence, these proposals will be argued to be either not well-defined, or to yield trivial gravitational energy.

Dewar and Weatherall ([15], fn. 30) enjoin such an examination of explicit proposals. It has two kinds of merits. After all, in empirically equivalent theories, radically different objects can play the same role. (Think of Starobinski's original model of cosmic inflation (see, e.g., [11], Sects. 2, 3 for details.) In one formulation, the latter is driven by a scalar, hence arguably a matter field on spacetime. In an equivalent formulation, inflation is merely a manifestation of spacetime curvature deviating from what it should be according to GR). Furthermore, comprehending the various possibilities in which a conceptually rich theory such as NCT can fail to exhibit a certain feature considerably enhances our understanding of it. In particular, this broadening of our repertoire of instruments is likely to pay off in comparing NCT to other theories in its theoretical vicinity, such as GR. (In the apt terms of Pitts [52]: Spacetime philosophy should aspire to "modal cosmopolitanism"-rather than "modal provincialism".) 


\subsubsection{Pseudotensors}

In this subsection, we evaluate the natural NCT counterparts of the general-relativistic pseudotensors as possible candidates for gravitational energy. They are found to trivialise the latter.

The standard approach to gravitational energy in GR proceeds via the Noether theorems. ${ }^{23}$ The absence of a (tractable, natural) Lagrangian or Hamiltonian formulation of NCT encumbers this road, though. ${ }^{24}$ It's straightforward to find a Lagrangian with suitable multipliers. But the latter are, of course, under-determined. Canonical gravitational energy-momentum for (i.e. the Noether current attributed to) the (non-flat) NCT metric would depend on the Lagrange multipliers, and hence would be ill-defined.

One might, however, take the definitions of pseudotensors, as familiar from GR, and just stipulate their formal NCT analogues. What encourages such a procedure is that pseudotensors - at least in GR - arguably satisfy natural desiderata for local gravitational energy, e.g. a conservation law, the dependence only on first derivatives of the field variables, or the reduction to the familiar Newtonian potential energy in the weak-field limit ([17], Sect. 3.2).

Following Goldberg [26], an infinitely large class of pseudotensor densities (of arbitrary weights $n+1, n \in \mathbb{N} \geq 0$ ) can be constructed as follows. (We restrict ourselves to mixed indices - one up, one down.)

$$
\vartheta_{\mu}^{(n) v}=|g|^{\frac{n}{2}}\left\{\vartheta_{\mu}^{v}+\frac{n}{2} U_{\mu}^{[v \sigma]} \partial_{\sigma} \ln |g|\right\}
$$

Here, $|g|$ denotes the modulo of the determinant of GR's metric. $U_{\mu}^{[\nu \sigma]}$ denotes a so-called super-potential. (The details needn't detain us here.)

For $n=0$, we obtain the weight-one density of the Einstein-pseudotensor $t_{\mu}^{\nu 25}$ :

$$
\vartheta_{\mu}^{(0) \nu}=\sqrt{|g|} t_{\mu}^{\nu}:=2 \sqrt{|g|} G_{\mu}^{\nu}+\partial_{\sigma}\left(|g|^{-\frac{1}{2}} g_{\mu \lambda} \partial_{\rho}\left(|g| g^{\lambda[v} g^{\sigma] \rho}\right)\right) .
$$

Together with the matter energy-momentum tensor $|g|^{\frac{n+1}{2}} T_{v}^{\mu}$, (of weight $n+1$ ), the pseudotensors-representing gravitational energy-momentum-form the system's total energy-momentum $\mathcal{T}_{\mu}^{(n) v}:=|g|^{\frac{n+1}{2}} T_{\nu}^{\mu}+\vartheta_{\mu}^{(n) v}$. The latter satisfies the continuity equation:

$$
\partial_{\nu} \mathcal{T}_{\mu}^{(n) v}=0
$$

Albeit not a tensor equation, this continuity equation holds in all coordinate systems. Hence, total energy-momentum can be said to be (locally/differentially) conserved.

For the NCT counterparts to the general-relativistic pseudotensors, it's tempting to replace the general-relativistic metric in the above expressions by NCT's spatial or

\footnotetext{
23 Historically too, this was Einstein's route-avant la lettre (Brading [5]).

24 In private correspondence, Nic Teh has conjectured that the non-existence of a Lagrangian or Hamiltonian formulation without Lagrange multipliers of NCT is even provable (cf. [28]).

25 Alternate formulations can be found in e.g. Dirac ([16], Ch. 31, 32) or Ohanian and Ruffini ([49], A5).
} 
temporal pseudo-metric, $h^{a b}$ and $t_{a b}=t_{a} t_{b}$ respectively. In fact, it can be shown [3] that NCT doesn't admit of a non-degenerate metric with which the NCT connection is compatible. Hence, the subsequent discussion is without loss of generality.

However, due to their degeneracy, i.e. vanishing determinant, this is a non-starter: One can easily verify that the resulting NCT pseudotensors either are trivial or nor defined at all. The latter is the case for $\vartheta_{\mu}^{(0) \nu}$, i.e. $n=0^{26}$; the former is the case for $\vartheta_{\mu}^{(n) v}$ s for $n>0$.

In conclusion: The natural NCT counterparts to GR's standard pseudotensor weights either are either ill-defined, or they yield a trivial notion of gravitational energy. While consonant with Dewar and Weatherall's conclusions, this result has nothing to do with a lack of gauge-invariance. ${ }^{27}$

\subsubsection{Komar Mass}

This section is devoted to a plausible definition of total energy of NCT spacetimes via the Komar integral. Like pseudotensors, it trivialises gravitational energy.

The most natural path to a global notion of gravitational energy in GR proceeds via the Noetherian route or, equivalently, the Hamiltonian formalism. As mentioned in the preceding section, for NCT this path is blocked. For static spacetimes in GR, an alternative exists: the Komar integral. (In GR, it coincides with the Hamiltonian definition, see e.g. [53], Ch. 4.3.)

Consider a static spacetime, i.e. one with a(n asymptotically normalised) time-like Killing field $\xi$, satisfying $\nabla_{(a} \xi_{b)}=0$. For such a spacetime, there exists a natural definition of "holding an object in place" via $\xi$ 's orbit (see [71] for details). This gives rise to a likewise natural notion of acceleration with respect to this orbit. Via this acceleration, a force can be defined that an observer at infinity must exert in order to keep a unit mass in place. Analogously to the characterisation of the total energy of the electrostatic field in terms of its asymptotic properties, we thus arrive-after various manipulations, for which we refer to the literature (ibid.) —at the following expression for the energy enclosed in the topological 2-sphere $\mathcal{S}_{t}$ in the hypersurface orthogonal to $\xi$ :

$$
E=-8 \pi \lim _{\mathcal{S}_{t} \rightarrow \infty} \oint_{\mathcal{S}_{t}} d \sigma^{a b} \nabla_{a} \xi_{b} .
$$

Here, $d \sigma^{a b}$ denotes the surface element on $\mathcal{S}_{t}$. This integral can serve as a definition of total energy in general-relativistic static spacetimes. It turns out to be conserved.

\footnotetext{
${ }^{26}$ Dirac's affine form of the weight-1 Einstein pseudotensor density also yields a vanishing result for a singular metric.

27 GR's pseudotensors are usually regarded as tainted by the problem of coordinate dependence (cf., for instance, Weyl [77], p. 273). By contrast, NCT's pseudotensors are free from that evil: Whenever they are defined, the NCT pseudtensor densities vanish coordinate-independently. In the same vein, they are-albeit trivially—invariant under dynamical symmetries.
} 
Given that NCT spacetimes are static in a natural sense, ${ }^{28}$ it's now tempting to stipulate the NCT counterpart of the Komar integral as a candidate for the total energy of NCT spacetimes as well. To that end, one plausibly replaces the Killing field in the Komar expression's integrand by NCT's time covector, $\xi_{a} \rightarrow t_{a}$. This already suffices to trivialise the proposal: Due to the compatibility condition of NCT's time pseudometric, $\nabla_{a} t_{b}=0$, the NCT counterpart of the Komar integral vanishes. Consequently, the total energy of a NCT spacetime would be zero. Gravitational energy-understood as the energy left after subtracting the energy contributions of ordinary matter-would then always exactly counterbalance matter energy. This is implausible for reasons that we'll explain in the next subsection, in which we'll discuss Lorentz and Levi-Civita's proposal.

\subsubsection{Lorentz and Levi-Civita's Proposal}

Lorentz and Levi-Civita proposed the Einstein tensor, $\mathrm{G}_{a b}=\mathrm{R}_{a b}-\frac{1}{2} R \mathrm{~g}_{a b}$ (or, for reasons of dimensionality, $-\frac{1}{2 \kappa} \mathrm{G}_{a b}$, with $\kappa:=\frac{4 \pi G}{c^{4}}$ ) as a representation of gravitational energy in GR (for details, see [8], Sects. 5-11). Is this convincing for the NCT case? For reasons again both general and specific to NCT, we argue that this isn't the case.

Three facts commend Lorentz and Levi-Civita's proposal. (1) In contrast to pseudotensorial approaches, the Einstein tensor is a bona fide tensor. (2) It obeys a bona fide covariant conservation law: the contracted Bianchi identity, $\nabla_{b} G^{a b} \equiv 0$. The attendant total energy-momentum ${ }_{(L L C)} \mathfrak{T}^{a b}:=-\frac{1}{2 \kappa} G^{a b}+\mathrm{T}^{a b}$, satisfies both an ordinary and covariant continuity equation, $\partial_{b}\left((L L C) \mathfrak{T}^{a b}\right)=\nabla_{b}\left((L L C) \mathfrak{T}^{a b}\right)=0$. (3) The Einstein tensor is the exact gravitational counterpart of the matter energy-momentum tensor: Whereas the latter is defined variationally as $T_{a b}=-\frac{2}{\sqrt{|g|}} \frac{\delta}{\delta g^{a b}}\left(\sqrt{|g|} \mathcal{L}_{(m)}\right)$, one obtains the Einstein tensor (up to a proportionality factor) by replacing the matter Lagrangian by the purely gravitational Einstein-Hilbert Lagrangian,

$$
G_{a b} \propto \frac{1}{\sqrt{|g|}} \frac{\delta}{\delta g^{a b}}(\sqrt{|g|} R) .
$$

The first two features carry over to NCT. The third one, however, doesn't: The absence of a natural Lagrangian formulation of NCT's full gravitational sector (cf. [28]) — including the two Trautmann conditions imposed on curvature-weakens the analogy between the Einstein tensor and the matter energy-momentum tensor.

But there are stronger reasons to question Lorentz and Levi-Civita's proposal: physical implausibility and vacuity, respectively (cf. [50], fns 180-181). Firstly, consider the Einstein Equations in vacuum. This, on Lorentz and Levi-Civita's proposal, yields vanishing gravitational energy, $G_{a b}=0$. But that's counterintuitive: Since the Einstein tensor is constructed from traces of the Riemann tensor, a solution of the vacuum Einstein Equations has in general non-vanishing Weyl structure. ${ }^{29}$ The latter encapsulates gravitational radiation. Prima facie, one would expect it to possess

\footnotetext{
28 That is: Its defining partial differential equations are elliptic. Hence, information about variations in a region propagates instantaneously.

29 Dewar and Weatherall ([15], Sect. 4) show that for NCT spacetimes, one can indeed define a (non-trivial) Weyl tensor (cf. [18, 20, 72]).
} 
gravitational energy - contrary to Lorentz and Levi-Civita's proposal (cf. [17] for a critique). Equally implausibly, it purports that there are no differences between gravitational energy in the exterior of a static and, say, rotating black hole, respectively: In either case, gravitational energy would be zero. For NCT, the objection needs to be slightly adapted. NCT's Poisson Equation is elliptic. Hence its solutions can't propagate. In that sense, there is of course no gravitational radiation. Still, one would expect different NCT spacetimes with non-vanishing Weyl structure-i.e. different homogenous solutions of the Poisson Equation - to differ in their gravitational energy. (Recall that the Weyl tensor measures tidal deformations in the shape of extended spacetime regions.)

Besides such doubts regarding its physical plausibility, it seems mysterious and contrived that, on Lorentz and Levi-Civita's proposal, any matter energy-momentum is exactly counterbalanced by gravitational energy (in the GR case): In all possible spacetimes, the total energy always vanishes, $-\frac{1}{2 \kappa} G_{a b}+2 \kappa T_{a b}=0$. It's elusive what positing such an entity would help explain. As Levi-Civita conceded in a letter to Einstein, the proposal is sterile in that "[...] the energy principle would lose all its heuristic value, because no physical process (or almost none) could be excluded a priori. In fact, [in order to get any physical process] one only has to associate with it a suitable change of the [gravitational field]". For NCT, this sterility is exacerbated by the fact that the Einstein tensor reduces to the Ricci tensor, and that the latter vanishes for mixed indices,

$$
{ }^{(N C T)} G_{a}^{b} \equiv{ }^{(N C T)} R_{a}^{b} \equiv 0 .
$$

In other words: Lorentz and Levi-Civita's proposal yields only a trivial gravitational energy-momentum flux along some direction $\xi^{a}:{ }^{(N C T)} G_{a}^{b} \xi^{a} \equiv 0$.

In conclusion: The Einstein tensor isn't suited for representing gravitational energy in both GR and NCT; it lacks physical informativeness and plausibility. The issue of gauge-dependence under dynamical shifts doesn't arise in any form.

Let's turn next to another tensorial proposal, Bel and Robinson's superenergy tensor.

\subsubsection{The Bel-Robinson Tensor}

In this subsection, we examine the NCT counterpart of the Bel-Robinson tensor as a candidate for NCT's gravitational energy.

Recall the energy-momentum tensor of electrodynamics:

$$
4 \pi T_{(e m)}^{\mu \nu}=F_{\lambda}^{\mu} F^{\lambda v}-\frac{1}{4} g^{\mu \nu} *\left(F^{\kappa \lambda}\right) *\left(F_{\kappa \lambda}\right)
$$

with the Faraday tensor $F^{\mu \nu}=\partial^{\mu} A^{\nu}-\partial^{\nu} A^{\mu}$, and its dual $*\left(F^{\mu \nu}\right)=\epsilon^{\mu \nu \kappa \lambda} F_{\kappa \lambda}$. (We use the latter-rather than the non-dual—in the second term of the energy-momentum tensor to render its structural similarity with the Bel-Robinson Tensor more transparent.) In an analogous manner, one can construct a tensor from the Riemann 
tensor, ${ }^{30}$ mimicking the symmetric electromagnetic energy-momentum tensor (see Garecki [22] for details). The result is the so-called "superenergy tensor":

$$
T^{a b c d}:=R^{a e f c} R_{e f}^{b d}+*\left(R^{a e f c}\right) *\left(R_{e f}^{b d}\right)=R^{a e f c} R_{e f}^{b d}+R^{a e f d} R_{e f}^{b d}-\frac{1}{2} g^{a b} R^{e f g c} R_{e f g}^{d} .
$$

(Here, $*$ denotes the usual dual operation: $*\left(R_{a b c d}\right)=\frac{1}{2} \epsilon_{a b e f} R_{c d}^{e f}$.) Bel and Robinson proposed it as a candidate for gravitational energy in GR.

As a consequence of the Bianchi identities (and hence, independently of the Einstein Equations), its covariant divergence vanishes:

$$
\nabla_{a} T^{a b c d} \equiv 0
$$

Note that due to the Einstein Equations, in vacuum the Riemann tensor can be replaced by the Weyl tensor. The latter encodes gravitational degrees of freedom that can propagate through vacuum. In light of this, the Bel-Robinson tensor seems apt for describing energy associated with gravitational radiation.

What makes it of particular interest is that the Bel-Robinson tensor appears in the expansion of the Einstein pseudotensor at a point, when evaluated in normal coordinates for some other point (see So, Nester and Chen [65] for details).

Due to the flatness of NCT spacetimes (in the sense of $R_{c d}^{a b}=0$, Sect. 3.1), a non-trivial Bel-Robinson tensor in NCT must be defined as a tensor of rank $(1,3)$ :

$$
{ }^{(N C T)} T_{k l m}^{i}:=R_{a b l}^{i} R_{m k}^{b}{ }^{a}+*\left(R_{a b l}^{i}\right) *\left(R_{m k}^{b}{ }^{a}\right),
$$

with the Riemann tensors (and their duals), associated with the NCT connection. As the Bianchi identities also hold in NCT, also $\nabla_{i}^{(N C T)} T_{k l m}^{i} \equiv 0$ obtains.

However, ${ }^{(N C T)} T_{k l m}^{i}$ isn't a convincing proposal for gravitational energy in NCT for reasons both general and specific to NCT.

Generally (and like in GR), it has the dimensions length ${ }^{-4}$. So, neither the BelRobinson tensor nor any of its powers have the right dimension, unless one introduces a novel constant of nature. But this seems ad-hoc.

Moreover, the Bel-Robinson tensor is linked to differences in pseudotensorial gravitational energy (and hence, on a standard interpretation of pseudotensors: to differences in gravitational energy simpliciter), rather than to the latter directly (ibid.). So, its physical interpretation would presuppose a non-trivial notion of pseudotensorial gravitational energy. But as we saw in Sect. 3.3.2, the most immediate NCT counterparts to pseudotensors are vacuous.

In conclusion: As a proposal for gravitational energy in NCT, the Bel-Robinson tensor is both formally, as well as in absence of its connection to non-vacuous pseudotensorial gravitational energy, unsuitable. Contra Dewar and Weatherall, gaugevariance isn't the issue here, though.

\footnotetext{
30 This may be motivated by Synge's suggestion that GR's gravitational field is represented by the Riemann curvature tensor (cf. [36] for a critical discussion). That is: According to Synge, one should view the Riemann tensor as the GR counterpart to the Faraday/field strength tensor-a view backed up by the perspective from the fibre bundle formalism [76].
} 
We conclude our perusal of candidates for gravitational energy in NCT with a non-tensorial proposal, due to Pitts.

\subsubsection{Pittsification}

Pitts [51] has recently propounded an astute solution to the problem of coordinate/gauge-dependence of pseudotensors in GR: Take your favorite pseudotensor, say the Einstein pseudotensor $\vartheta_{a}^{b}$, and declare the totality of its values in all possible coordinate systems (at neighbourhood of a point) one object. Symbolically:

$$
\left\{(\forall \text { coordinate systems CS })\left(\vartheta_{\mu}^{v}\right)_{C S}\right\} \text {. }
$$

It has (uncountably) infinite components. Each corresponds to the pseudotensor's value in one possible coordinate system.

There are two ways to transfer this idea to NCT. The first one takes the NCT counterparts of pseudotensors, and "Pittsifies" them as in Pitts' original proposal for GR. But this is of little interest, as the NCT counterparts of pseudotensors are either trivial or not defined (Sect. 3.3.1).

More auspicious is another option. It starts from $N G_{G S T}$ 's gravitational energy. As described in Sect. 3.2, a DPM in NCT $\mathfrak{M}$ can be de-geometrised into an equivalence class of GST models $G S T_{\alpha}(\mathfrak{M})$ for some index set $\alpha \in \mathcal{A}$. For any two $\alpha, \alpha^{\prime} \in \mathcal{A}$, the models $G S T_{\alpha}(\mathfrak{M})$ and $G S T_{\alpha^{\prime}}(\mathfrak{M})$ differ only up to dynamical shifts. Now Pittsify the gravitational energies of all these $G S T_{\alpha}(\mathfrak{M})$ s. This yields the (Pittsified) NCT gravitational energy, symbolically:

$$
E(\mathfrak{M}):=\left\{(\forall \alpha \in \mathcal{A}) E\left[G S T_{\alpha}(\mathfrak{M})\right]\right\}
$$

Each component of this object corresponds to one possible GST degeometrisation. By construction, it's gauge-invariant under dynamical shifts. (Recall: De-geometrisations of an NCT spacetime are all related via dynamical shifts.)

Pittsification welds together into one well-defined, formal object the gravitational energies of those GST spacetimes that correspond to the same NCT spacetime. It's not obvious, though, that it provides a satisfactory representation for gravitational energy in NCT: Firstly, its conceptual prerequisites seem alien to NCT; secondly, one may have qualms about its physical meaningfulness.

The Pittsified NCT gravitational energy is constructed from the gravitational energies of those $N G_{G S T}$ spacetimes the geometrisation of which yields the same NCT spacetime. On the one hand, this yields a formally well-defined object-even a geometric one. ${ }^{31}$ On the other hand, one may wonder: Is it legitimate to introduce into a theory quantities built from terms that belong to, and are meaningful only within, a different theory? That is: Are we allowed to use quantities prima facie intelligible only in $\mathrm{NG}_{\mathrm{GST}}$ in order to define a quantity supposedly meaningful in NCT?

\footnotetext{
31 If one is willing to extend the standard meaning of geometrical objects to objects with infinitely many components ([51], Sects. 1-2).
} 
Perhaps such a worry might be allayed by the thought that the individual degeometrised $\mathrm{NG}_{\mathrm{GST}}$ spacetimes lack meaning in NCT; only their totality accrues it. Consider the gauge-quantities of electromagnetism, the 4-potentials. By themselves, they don't possess physical significance, either (perhaps setting aside potential subtleties for the Aharonov-Bohm effect); only a suitable combination of them-i.e. the Faraday tensor-does. By analogy, one might argue that only the Pittsified NCT gravitational energy as a whole is meaningful; its individual components- the $\mathrm{NG}_{\mathrm{GST}}$ quantities-aren't. One could counter by questioning the whole procedure: Isn't Pittsification too cheap a trick to procure gauge-invariant quantities? Finding gaugeinvariant quantities is a formidable task in ongoing research in (non-Abelian) gauge theories. One would like more than a merely formal object: How to ensure that the Pittsified gravitational energy actually possesses physical significance ? $^{32}$ (Consider, by analogy, the Pittsification of the electromagnetic 4-potentials, i.e. the infinitecomponent object made up of all 4-potentials in all possible gauges. In a formal sense, it's evidently gauge-independent. One would baulk, however, at attributing it physical content, as expressed in the electromagnetic fields.)

In conclusion: Via Pittsification, we can define a formal candidate for gravitational energy of a NCT spacetime from the gravitational energies of its corresponding $\mathrm{NG}_{\mathrm{GST}}$ de-geometrisations. Reasons to object to this proposal don't include gauge-dependence; rather, they consist in doubts about its physical significance and conceptual adequacy. ${ }^{33}$

\section{Discussion}

Dewar and Weatherall [15] conclude their paper with "an important lesson for how to understand energy in geometrized theories. [...] (T)here is a deep relationship between the classical notions of energy, work, force, and inertia. Energy is a measure of the ability to do work [...] But in theories in which gravitation is 'geometrized' in the sense that gravitation is understood as an inertial effect in curved spacetime, we should not think of gravitation as a force at all —and so, in particular, it is not the sort of thing that does work. To the contrary, work makes sense only as a measure of the deviation from inertial motion over some distance."

Our discussion illustrates this insight in slightly more detail. ${ }^{34}$ It shows explicitly that non-geometrised variants of $\mathrm{NG}$ in the above sense- $-\mathrm{NG}_{\mathrm{NST}}$ and $\mathrm{NG}_{\mathrm{GST}}-$ do allow for a well-defined notion of gravitational energy. Contrariwise, for NG in spacetime settings where inertial structure has absorbed gravity-MHST and NCT--

\footnotetext{
32 This is squarely related to the question of inferences from symmetries to reality: Declaring the physical equivalence between symmetry-related models of a theory remains merely formal and verbal, unless a metaphysically perspicuous explication of the corresponding ontological picture is forthcoming [46].

33 This criticism mirrors the one mounted against Pittsification of pseudotensors in GR ([17], Sect. 3.3).

34 We plan to complement our and Dewar and Weatherall's results by an investigation from the viewpoint of teleparallisation (for a conceptual introduction, see [33]). Recently, Teh and Read [69] have shown that the Trautman Recovery Theorem is an instance of teleparallelisation. It will be interesting to study whether further illuminating insights into gravitational energy in NCT and NG can be gained by applying the machinery of teleparallelisation.
} 
gravitational energy faces several obstacles. ${ }^{35}$ The status of a prima facie central concept such as the energy associated with Newtonian gravitational degrees of freedom crucially depends on how Newtonian Gravity is interpreted.

Dewar and Weatherall make a farther-reaching suggestion: "[...] (W)e should understand the energy density of Yang-Mills fields [including electromagnetism, the authors] as relative to some background structure - namely, the inertial structure determined by the spacetime metric in general relativity" (p. 27, their emphasis). We concur with this. But it's worthwhile stressing that it implies a minor rectification of Dewar and Weatherall's conclusion, cited above: It's less the geometrisation of gravity itself-the absorption of gravitational effects by inertial structure - that is responsible for the difficulties in defining gravitational energy. Rather, it's the existence of (sufficiently rich) inertial structure simpliciter that seems to be a prerequisite for a meaningful (or at least, robust, cf. [56]) definition of field energies. ${ }^{36}$ In fact, one may construe the main problem diagnosed in Sect. 2.4 for gravitational energy for $\mathrm{NG}_{\mathrm{MHST}}$ as a violation of this requirement: MHST's inertial structure is too impoverished to even allow us to define gravitational energy; for that, the derivative operator had to be imported from GST.

Our discussion also emphasised an additional difficulty for gravitational energy for NCT: the absence of a natural Lagrangian (or Hamiltonian) formulation. Despite the similarities with respect to geometrizing gravity, this makes its status more precarious than in GR. Energy is arguably a cluster concept. But it wouldn't be too much of a stretch, either, to regard the definition of energy within the Lagrangian/Hamiltonian framework as the primary meaning of energy in field theories. Hence, we propose, not only will the comparison with Yang-Mills theories be rewarding, as Dewar and Weatherall suggest; it will likewise be illuminating to investigate the status of field energies in non-Lagrangian theories.

Open Access This article is distributed under the terms of the Creative Commons Attribution 4.0 International License (http://creativecommons.org/licenses/by/4.0/), which permits unrestricted use, distribution, and reproduction in any medium, provided you give appropriate credit to the original author(s) and the source, provide a link to the Creative Commons license, and indicate if changes were made.

\section{References}

1. Acuña, P.: Inertial trajectories in deBroglie-Bohm quantum theory: an unexpected problem. Int. Stud. Philos. Sci. 30(3), 201-230 (2016)

2. Anderson, J.L.: Principles of Relativity Physics. Academic Press, New York (1967)

3. Andringa, R., Bergshoeff, E., Panda, S., de Roo, M.: Newtonian gravity and the Bargmann algebra. Class. Quantum Gravity 28, 105011 (2011)

4. Belot, G.: An elementary notion of gauge equivalence. Gen. Relativ. Gravit. 40(1), 199-215 (2007)

\footnotetext{
35 It's important that the notion of geometrisation relevant here is specific-the absorption of gravitational effects into inertial structure. Other notions of geometrisation (see [37], Ch. 9) don't seem relevant. Reichenbach's geometrised toy unification of gravity and electromagnetism [23], for instance, admits of the standard GR electromagnetic energy-stress tensor.

36 This point seems pertinent for all theories in which the notion of inertial trajectories becomes questionable. In fact, this is the case for Bohmian Mechanics (see [1]): Energy is no longer a fundamental concept.
} 
5. Brading, K.: A note on general relativity, energy conservation, and Noether's theorems. In: Kox, A.J., Eisenthal, J. (eds.) The Universe of General Relativity. Birkhäuser, Basel (2005)

6. Brown, H.R.: Physical Relativity. Spacetime Structure from a Dynamical Perspective. Oxford University Press, Oxford (2005)

7. Bunge, M.: Energy: between physics and metaphysics. Sci. Educ. 9(5), 459-463 (2000)

8. Cattani, C., De Maria, M.: Conservation laws and gravitational waves in general relativity 1915-1918. In: Earman, J., et al. (eds.) The Attraction of Gravitation: New Studies in the History of General Relativity. Birkhäuser, Boston (1993)

9. Curiel, E.: Kinematics, Dynamics, and the Structure of Physical Theory (2016). http://philsci-archive. pitt.edu/11959/. Accessed 2 June 2018

10. Dasgupta, Sh: Symmetry—a metaphysical view. In: Knox, E., Wilson, A. (eds.) The Routledge Companion to Philosophy of Physics, p. 2018. Routledge, London (2018)

11. De Felice, A., Tsujikawa, S.: F(R) theories. Living Rev. Relat. (2010). https://doi.org/10.12942/lrr2010-3

12. Dewar, N.: Symmetries in Physics, Metaphysics and Logic. DPhil thesis, University of Oxford (2015)

13. Dewar, N. (2017). Sophistication about symmetries. The British Journal for the Philosophy of Science, Vol. 70, Issue 2, pp 485-521

14. Dewar, N. (2018). Maxwell Gravitation. Philosophy of Science 85:249

15. Dewar, N. \& Weatherall, J. (2018). On gravitational energy in Newtonian theories. Foundations of Physics Vol. 48, pp. 558-578

16. Dirac, P.A.M.: The General Theory of Relativity. Princeton University Press, Princeton (1975)

17. Dürr, P. (2018). Fantastic Beasts and where (not) to find them: local gravitational energy and energy conservation in general relativity. Studies in History and Philosophy of Modern Physics 65:1-14

18. Duval, C., Gibbons, G., Horvathy, P.: Conformal and projective symmetries in Newtonian cosmology. J. Geom. Phys. 112, 197-209 (2017)

19. Earman, J.: World Enough and Spacetime. MIT Press, Cambridge (1989)

20. Ehlers, J., Buchert, T.: On the Newtonian limit of the Weyl tensor. Gen. Relativ. Gravit. 41(9), 2153-2158 (2009)

21. Friedman, M.: Foundations of Space-Time Theories. Relativistic Physics and Philosophy of Science. Princeton University Press, Princeton (1983)

22. Garecki, J.: Some Remarks on the Bel-Robinson Tensor (2018). https://arxiv.org/abs/gr-qc/0003006. Accessed 20 July 2018

23. Giovanelli, M.: 'But I still can't get rid of a sense of artificiality' - the Reichenbach-Einstein debate on the geometrization of the electromagnetic field. Stud. Hist. Philos. Mod. Phys. 54, 35-51 (2016)

24. Glymour, C.: Theoretical realism and theoretical equivalence. In: Buck, R.C., Cohen, R.S. (eds.) Boston Studies in the Philosophy of Science, vol. 8, p. 1971. Reidel, Dordrecht (1970)

25. Glymore, C. (1977). Indistinguishable space-times and the fundamental group. Minnesota Studies, vol. 8 (25):pp 50-60

26. Goldberg, J.N. (1958). Conservation laws in general relativity. Physical Review 111, 315

27. Greaves, H. \& Wallace, D. (2014). Empirical consequences of symmetries. British Journal for the Philosophy of Science 65 (1): 59-89

28. Hansen, D., Hartong, J., Obers, N.: Action principle for Newtonian gravity. Phys. Rev. Lett. 122, 061106 (2019)

29. Hoefer, C.: The metaphysics of space-time substantivalism. J. Philos. 93(1), 5-27 (1996)

30. Hoefer, C., Huggett, N.: Absolute and relational theories of space and motion. Stanf. Encycl. Philos. (2017). https://plato.stanford.edu/entries/spacetime-theories/. Accessed 2 May 2018

31. Huggett, N.: Why manifold substantivalism is probably not a consequene of classical mechanics. Int. Stud. Philos. Sci. 13, 17-34 (1999)

32. Ismael, J.: Symmetry and superfluous structure. In: Knox, E., Wilson, A. (eds.) The Routledge Companion to Philosophy of Physics, p. 2018. Routledge, London (2018)

33. Knox, E. (2011). Newton-Cartan theory and teleparallel gravity: the force of a formulation. Studies in History and Philosophy of Modern Physics, Vol. 42, Issue 4, Pages 264-275

34. Knox, E.: Newtonian spacetime structure in light of the equivalence principle. Br. J. Philos. Sci. 65(4), 863-880 (2014)

35. Knox, E.: Physical relativity from a functionalist perspective. Stud. Hist. Philos. Mod. Phys. 67, 118-124 (2017) 
36. Lehmkuhl, D.: Is spacetime a gravitational field? In: Dieks, D. (ed.) The Ontology of Spacetime II. Philosophy and Foundations of Physics, vol. 4, pp. 83-110. Elsevier, Amsterdam (2008)

37. Lehmkuhl, D.: Spacetime Matters. DPhil thesis, University of Oxford (2008b)

38. Lehmkuhl, D.: Why Einstein did not believe that general relativity geometrizes gravity. Stud. Hist. Philos. Mod. Phys. 46, 316 (2014)

39. Malament, D.: Topics in the Foundations of General Relativity and Newtonian Gravitation Theory. University of Chicago Press, Chicago (2012)

40. Martens, N., Lehmkuhl, N.: Dark Matter = Modied Gravity? Scrutinising the spacetime-matter distinction through the modied gravity/dark matter lens. Unpublished manuscript (ms)

41. Martens, N., Read, J.: Sophistry about symmetries? Unpublished manuscript (ms)

42. Maudlin, T. (1988). The essence of spacetime. In: PSA: Proceedings of the Biennial Meeting of the Philosophy of Science Association, Volume Two: Symposia and Invited Papers

43. Maudlin, T.: Philosophy of Physics: Space and Time. University of Oxford Press, Oxford (2012)

44. Misner, Ch., Thorne, K., Wheeler, A.: Gravitation. W. H. Freeman, San Fransisco (1973)

45. Møller-Nielsen, T.: Symmetry, Indiscernibility, and the Generalist Picture. DPhil thesis, University of Oxford (2015)

46. Møller-Nielsen, Th: Invariance, interpretation and motivation. Philos. Sci. 84(2017), 1253-1264 (2017)

47. Nerlich, G.: Einstein's Genie: Spacetime Out of the bottle. Minkowski Institute Press, Montréal (2013)

48. Norton, J.: What can we learn about the ontology of space and time from the theory of relativity? In: Sklar, L. (ed.) Physical Theory: Method and Interpretation, p. 2014. Oxford University Press, Oxford (2014)

49. Ohanian, H., Ruffini, R.: Gravitation and Spacetime. Cambridge University Press, Cambridge (2013)

50. Pauli, W.: Theory of Relativity. Dover, New York (1981)

51. Pitts, B.: Gauge-invariant localization of infinitely many gravitational energies from all possible auxiliary structures. Gen. Relativ. Gravit. 42(601-622), 2010 (2010)

52. Pitts, B.: Space-time constructivism vs. modal provincialism; or, how special relativistic theories needn't show Minkowski geometry. Stud. in Hist. and Philos. of Mod. Phys., Special Issue on Harvey Brown's "Physical Relativity 10 years later" (2017)

53. Poisson, E.: A Relativist's Toolkit: The Mathematics of Black Hole Mechanics. Cambridge University Press, Cambridge (2004)

54. Pooley, O.: Substantivalist and relationalist approaches to spacetime. In: Batterman, R. (ed.) The Oxford Handbook of Philosophy of Physics, p. 2012. Oxford University Press, Oxford (2013)

55. Quine, W.O.: Ontology and ideology. Philos. Stud. 2(1), 11-15 (1951)

56. Read, J.: Functional gravitational energy. Br. J. Philos. Sci. (2017). https://doi.org/10.1093/bjps/axx048

57. Read, J. \& Møller-Nielsen, T. (2018). Motivating dualities. Synthese (forth.)

58. Rey, D.: Similarity Assessments, Spacetime, and the Gravitational Field: What Does the Metric Tensor Represent in General Relativity (2013). http://philsci-archive.pitt.edu/9615/

59. Rohrlich, F.: Classical Charged Particles. World Scientific, Singapore (2007)

60. Rovelli, C.: Halfway through the woods: contemporary research on space and time. In: Earman, J., Norton, J. (eds.) The Cosmos of Science, pp. 180-223. University of Pittsburgh Press, Pittsburgh (1997)

61. Rovelli, C.: Why gauge? Found. Phys. 44(2014), 91-104 (2014)

62. Rynasiewicz, R.: Absolute versus relational space-time: an outmoded debate?'. J. Philos. 93(6), 279-306 (1996)

63. Rynasiewicz, R.: Newton's view on space, time and motion. Stanf. Encycl. Philos. (2011). https:// plato.stanford.edu/entries/newton-stm/

64. Saunders, S.: Rethinking Newton's 'principia'. Philos. Sci. 80(1), 22-48 (2013)

65. So, L.L., Nester, J., Chen, H.: Energy-momentum density in small regions: the classical pseudotensors. Class. Quantum Gravity 26(085004), 2009 (2009)

66. Sotiriou, T., Faraoni, V. \& Liberati, S.: Theory of Gravitational Theories. A No Progress Report (2008). https://arxiv.org/pdf/0707.2748.pdf

67. Szabados, L.: Quasi-local energy-momentum and angular momentum in general relativity. Living Rev. Relativ. 12(4), 2009 (2009)

68. Teh, N.: Recovering Recovery: On the relationship between gauge symmetry and Trautman Recovery (2017). http://philsci-archive.pitt.edu/13297/. Accessed 10 Sept 2018

69. Teh, N., Read, J.: The teleparallel equivalent of Newton-Cartan gravity. Class. Quantum Gravity 35, 18 (2018) 
70. Trautman, A.: Foundations and current problems of General relativity-Brandeis summer institute in theoretical physics. In: Deser, S., Ford, K.W. (eds.) Lectures on General Relativity, pp. 1-248. Prentice Hall, Englewood Cliffs (1965)

71. Wald, R.: General Relativity. University of Chicago Press, Chicago (1984)

72. Wallace, D.: More problems for Newtonian cosmology. Stud. Hist. Philos. Mod. Phys. 57, 55 (2016)

73. Weatherall, J.: Maxwell-Huygens, Newton-Cartan, and Saunders-Knox Spacetimes. Philos. Sci. 83(1), 82-92 (2015)

74. Weatherall, J.: Space, Time, and Geometry from Newton to Einstein, feat. Maxwell. (lecture notes, circulated at MCMP Summerschool on Mathematical Philosophy, 2016) (2016a)

75. Weatherall, J.: Are Newtonian gravitation and geometrized Newtonian gravitation theoretically equivalent? Erkenntnis 81(5), 1073-1091 (2016)

76. Weatherall, J.: Fiber bundles, Yang-Mills theory, and General Relativity. Synthese 193(8), 2389-2425 (2016)

77. Weyl, H.: Raum, Zeit, Materie. Vorlesungen über allgemeine Relativitästheorie. Springer, Berlin (1923)

Publisher's Note Springer Nature remains neutral with regard to jurisdictional claims in published maps and institutional affiliations. 